\title{
Synthesis and Characterization of 3-Methoxypropyldithiocarbamate Complexes with Iron(II), Cobalt(II), Nickel(II), Copper(II) and Zinc(II) and Their Adducts with Nitrogen Base Ligands
}

\author{
Saad E. Al-Mukhtar \\ Marwa Th. Aghwan \\ Department of Chemistry \\ College of Science \\ University of Mosul
}

(Received 13/9/2012;Accepted 17/12/2012)

\begin{abstract}
New complexes of dithiocarbamate of the general formula [M(3-MeoPrdtc $\left.)_{2}\right]$ and [M(3-MeoPrdtc) $\left.)_{2} \cdot \mathrm{nL}\right]$ Where $\mathrm{M}=\mathrm{Fe}(\mathrm{II}), \mathrm{Co}(\mathrm{II}), \mathrm{Ni}(\mathrm{II}), \mathrm{Cu}(\mathrm{II})$ and $\mathrm{Zn}(\mathrm{II})$, and (3-MeoPrdtc) =3-Methoxypropyldithiocarbamate, and $\mathrm{n}=2$ when $\mathrm{L}=$ pyridine, Isoquinoline, $\gamma$-picoline, 3,5-lutidine, $\mathrm{n}=1$ when $\mathrm{L}=$ ethylenediamine, $(1,10)$-phenanthroline, have been prepared and characterized by metal analyses, infrared, conductance measurements, electronic absorption spectra and suscetptibility measurements. Magnetic moment and electronic spectra, indicate that the complexes of the type $\left[\mathrm{M}(3 \text {-meoprdtc })_{2}\right]$ are of tetrahedral geometry while the complexes of the type $\left[\mathrm{M}(3-\text { meoprdtc })_{2} \mathrm{~nL}\right]$ have octahedral geometry.
\end{abstract}

Keywords: Dithiocarbamate, Iron(II), Cobalt(II), Nickel(II), Copper(II), Zinc(II), Complexes.

\section{تخضير وتشخيص معقدلت لليكلن 3 تميثوكسي برويل شنائي ثليوكاربميت مع الحسيد(II)، الكوبل(II)، النيل(III)، النحس)(II) والخارصبن(II) وعددمن الفواعد النتروجينية}

\section{الملغص}

حضرت معقدات جديدة ذات صيغة [M(3-MeoPrdtc) 2وذلك بمفاعلة الليكاند (3-MeoPrdtc)

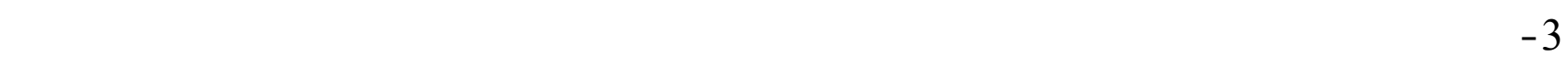

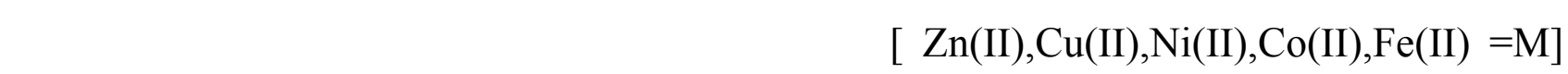

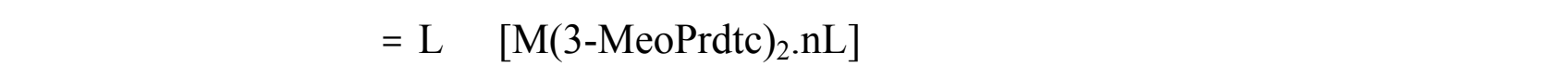

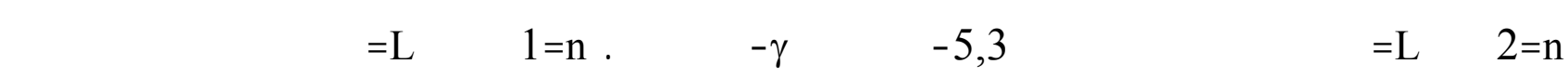

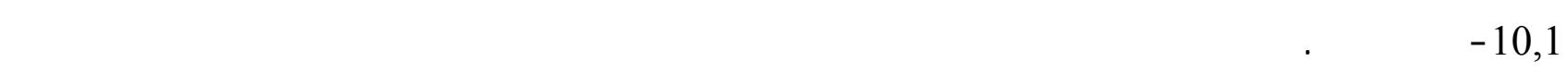

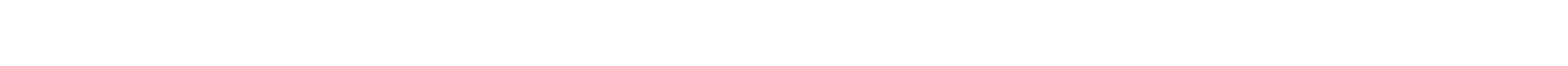
الالكتروني على اتخاذشكل رباعي اللطوح للمعقدات ذات الصيغة [M(3-MeoPrdtc) في حين المعقدات اللسلسية التنلسق ذات الصيغة [M(3-MeoPrdtc)2.nL] التخذتشكل ثماني اللنطوح. 


\section{INTRODUCTION}

The bis- (dithiocarbamate) complexes $\left[\mathrm{M}\left(\mathrm{S}_{2} \mathrm{CNR}_{2}\right)_{2}\right]$, where $\mathrm{R}=$ alkyl group, are the most common among other ligand and complexes. Many studies and reviews have been published on transition and non-transition metals (Onwudiwe and Ajibade, 2010) which indicate that there are a wide variety of compounds of anions such as dithiocarbamate, dithiocarbonate, dithiocarboxylate, trithiocarbonate, xanthate, thioxanthate, dithiophosphinate and dialkyldithiophosphinate (Rathore et al., 2007).

Dithiocarbamate and dithiophosphinate complexes have important uses. Dithiocarbamates derived from secondary amines are the most studied ones because they are stable and possess interesting electrochemical and optical properties (Cotero-Villegas et al., 2010). Most of aliphatic and aromatic dithiocarbamates synthesized until now may coordinate only through the dithio-group, behaving as uninegative bidentate ligands in reactions with metal ions (Leka et al., 2006).

Transition metal dithiocarbamate complexes find use in diverse applications such as material science, medicine and agriculture (Shaheen et al., 2007) and possess interesting structural chemistry (Onwudiwe and Ajibade, 2011) that make their study continuously attractive, and biological activity (as antibacterial, cytostatic, antifungal and immunoregulatory) most of these applications are based on the complexation properties of dithiocarbamate ligands with metal ions, especially with transition metal ions (Golcu, 2006).

In view of these interesting results, we have prepared a new dithiocarbamate ligand and its complexes with different metal ions and their nitrogeneous adducts.

\section{Materials and Methods}

\section{EXPERIMENTAL}

All reagents and solvents were of analytical grade and used as supplied from Fluka or BDH chemical companies. Infrared spectra were recorded on a Brucker Tensor 27co. FTIR spectrophotometer in the $400-4000 \mathrm{~cm}^{-1}$ range using $\mathrm{KBr}$ discs. Conductivity measurements were carried out on a $10^{-3} \mathrm{M}$ solution of the complexes in DMF using conductivity meter PCM3 Jenway at ambient temperature. The electronic spectra were recorded on a Shimadzu UV-visible spectrophotometer UV-160 for $10^{-3} \mathrm{M}$ solution of complexes in DMF as solvent at $25^{\circ} \mathrm{C}$ using $1 \mathrm{~cm}$ quartz cells Metals content were determined using AA670 atomic absorption. Melting points were recorded on an Electrothermal 9300 apparatus and were uncorrected. The magnetic measurement was carried out at $25^{\circ} \mathrm{C}$ on the solids by Faraday's method using Brucker BM6 instrument.

\section{Synthesis of potassium 3-Methoxypropyldithiocarbamate}

To $(8.90 \mathrm{~g}, 0.10 \mathrm{~mol})$ of 3-Methoxypropylamine was added to aqueous solution $(5.61 \mathrm{~g}, 0.10 \mathrm{~mol})$ of potassium hydroxide with stirring. The mixture was cooled in an ice bath, to this mixture $\left(7.60 \mathrm{~cm}^{3}, 0.10 \mathrm{~mol}\right)$ of carbon disulfide was added dropwise with continuous stirring for $30 \mathrm{~min}$ in ice bath. The precipitate formed was extracted with $(100 \mathrm{ml})$ diethylether filtered off, washed with diethylether and dried under vacuum. A white precipitate formed. 


\section{I.A. Synthesis of complex [M (3-MeoPrdtc) $)_{2}$ ] \\ $\mathrm{M}=\mathrm{Fe}(\mathrm{II}), \mathrm{Co}(\mathrm{II}), \mathrm{Ni}(\mathrm{II}), \mathrm{Cu}(\mathrm{II}), \mathrm{Zn}(\mathrm{II})$}

Ethanolic solution of $\mathrm{FeCl}_{2} \cdot \mathrm{XH}_{2} \mathrm{O}(1.26 \mathrm{~g}, 0.01 \mathrm{~mol})$ or $\mathrm{CoCl}_{2} \cdot 6 \mathrm{H}_{2} \mathrm{O}(2.37 \mathrm{~g}, 0.01 \mathrm{~mol})$ or $\mathrm{NiCl}_{2} .6 \mathrm{H}_{2} \mathrm{O}(2.37 \mathrm{~g}, 0.01 \mathrm{~mol})$ or $\mathrm{CuCl}_{2} .2 \mathrm{H}_{2} \mathrm{O}(1.70 \mathrm{~g}, 0.01 \mathrm{~mol})$ or $\mathrm{ZnCl}_{2}(1.36 \mathrm{~g}, 0.01$ mol) was added dropwise to ethanolic solution of potassium 3methoxypropyldithiocarbamate $(4.06 \mathrm{~g}, 0.02 \mathrm{~mol})$ with stirring for 30 min until complete precipitation. The precipitate was filtered off, washed with ethanol. Then with diethylether and dried under vacuum.

\section{I.B. Synthesis of complex [M (3-MeoPrdtc $\left.)_{2}(\mathrm{~L})_{2}\right]$ $\mathrm{L}=$ pyridine, isoquinoline, $\gamma$-picoline, 3,5 -lutidine}

It was prepared similarly as in (I.A.). The precipitate formed was treated with $(0.02 \mathrm{~mol})$ of (pyridine, isoquinoline, $\gamma$-picoline, 3,5-lutidine) dropwise with continuous stirring for $30 \mathrm{~min}$, the precipitate formed, was filtered and washed with ethanol then dried under vacuum.

\section{I.C. Synthesis of complex [M (3-MeoPrdtc $\left.)_{2}(\mathrm{~L})\right]$}

\section{$L=1,10$-phenanthroline, ethylenediamine}

It was prepared similarly as in (I.A.) and the precipitate formed was treated with (0.01 mol) of (1,10-phenanthroline,ethylenediamine) dropwise with continuous stirring for 30 min, the precipitate formed was, filtered, washed with ethanol, then dried under vacuum.

\section{RESULTS AND DISCUSSTION}

The new ligand was prepared by the reaction of 3-methoxypropylamine with potassium hydroxide and then added carbon disulphide: the complexes were prepared through direct reaction of the metal chlorides, $\mathrm{FeCl}_{2} \cdot \mathrm{XH}_{2} \mathrm{O}$ or $\mathrm{CoCl}_{2} \cdot 6 \mathrm{H}_{2} \mathrm{O}$ or $\mathrm{NiCl}_{2} \cdot 6 \mathrm{H}_{2} \mathrm{O}$ or $\mathrm{CuCl}_{2} \cdot 2 \mathrm{H}_{2} \mathrm{O}$ and $\mathrm{ZnCl}_{2}$.unhydrous with the above ligand in (1:2) molar ratio. The values of conductivity in dimethylformamide solution of the complexes range from (0.40-25.10) $\Omega^{-1} . \mathrm{cm}^{2} . \mathrm{mol}^{-1}$, which are typical values for non electrolyte type (Geary, 1971).

\section{Infra-red spectral studies}

The important IR bands of the ligand and its complexes are listed in Table (2). The stretching frequency of the $v(C-\ldots)$ band for the dithiocarbamates was intermediate between the stretching frequencies associated with typical single and double-bonded carbon and nitrogen atoms (Srinivasan et al., 2010). The $v(\mathrm{C}-\mathrm{N})$ and $v(\mathrm{C}-\mathrm{S})$ were observed in the ranges of (1500-1550) $\mathrm{cm}^{-1}$ and $(968-1038) \mathrm{cm}^{-1}$. The presence of only one band in the later region reports the bidentate coordination of the dithio ligand (Serrano, 2003).

IR spectra showed a new band at (418-445) $\mathrm{cm}^{-1}$ which is the evidence for the coordination of metal to sulfur $v(\mathrm{M}-\mathrm{S})$. This behavior may be attributed to the electronreleasing of the amines, which forces high electron density towards the sulfur atoms, the $v(\mathrm{M}-\mathrm{N})$ was observed in the $(465-523) \mathrm{cm}^{-1}$ region (Raya et al., 2006). In the adduct, the band corresponding to $v(\mathrm{~N}-\mathrm{H})$ of the ethylenediamine $(6,13,21$ and 27) was observed at lower frequency $(3211-3270) \mathrm{cm}^{-1}$ (Montagner et al., 2011). This indicates that it was shown in coordination with metal ions, as well as the band $v(\mathrm{C}=\mathrm{N})$ ring which was 
observed as (1441-1522) $\mathrm{cm}^{-1}$ which means the coordination of the donor atoms with the metal ios (Geraldo et al., 2011).

\section{Electronic spectral studies}

The UV-Visible spectra of the ligands and their complexes of $10^{-3} \mathrm{M}$ solution in DMF were recorded; the results were listed in Table (2). the UV-Visible spectrum of the Fe(II) complex (1) gives an absorption band at $\left(11180 \mathrm{~cm}^{-1}\right)$, which corresponds to $\left({ }^{5} \mathrm{E} \rightarrow{ }^{5} \mathrm{~T}_{2}\right)$ transition in a tetrahedral geometry (Nicholls, 1973). Complexes (2-7) show absorption bands at the range (9803-11095 $\left.\mathrm{cm}^{-1}\right)$, which were assigned $\operatorname{to}\left({ }^{5} \mathrm{~T}_{2} \mathrm{~g} \rightarrow{ }^{5} \mathrm{Eg}\right)$ transition (Coucouvanis and Fackler, 1967).

The Co(II) complex (8) exhibited an absorption band at $\left(15673 \mathrm{~cm}^{-1}\right)$ region, which was assigned $\left({ }^{4} \mathrm{~A}_{2}(\mathrm{~F}) \rightarrow{ }^{4} \mathrm{~T}_{1}(\mathrm{P})\right)$ transition (Siddiqi and Nishat.; 2000)., in tetrahedral configuration of this complex and the absence of $\left({ }^{4} \mathrm{~A}_{2}(\mathrm{~F}) \rightarrow{ }^{4} \mathrm{~T}_{2}(\mathrm{~F})\right)$ and $\left({ }^{4} \mathrm{~A}_{2}(\mathrm{~F}) \rightarrow{ }^{4} \mathrm{~T}_{1}(\mathrm{~F})\right)$, are due to the sensitivity of the instrument used. Complexes (9-14) show three absorption bands at the range (9700-9842 $\left.\mathrm{cm}^{-1}\right),\left(15243-16891 \mathrm{~cm}^{-1}\right)$ and $\left(20716-25252 \mathrm{~cm}^{-1}\right)$, which were assigned to $\left({ }^{4} \mathrm{~T}_{1} \mathrm{~g}(\mathrm{~F}) \rightarrow{ }^{4} \mathrm{~T}_{1} \mathrm{~g}(\mathrm{~F})\right),\left({ }^{4} \mathrm{~T}_{1} \mathrm{~g}(\mathrm{~F}) \rightarrow{ }^{4} \mathrm{~A}_{2} \mathrm{~g}(\mathrm{~F})\right)$ and $\left({ }^{4} \mathrm{~T}_{1} \mathrm{~g}(\mathrm{~F}) \rightarrow{ }^{4} \mathrm{~T}_{1} \mathrm{~g}(\mathrm{P})\right)$ transition in octahedral configuration (Martel, 1971).

The Ni(II) complex (15) shows two absorption bands at $\left(10066 \mathrm{~cm}^{-1}\right)$ and $\left(13022 \mathrm{~cm}^{-1}\right)$ which were assigned to $\left({ }^{3} \mathrm{~T}_{1}(\mathrm{~F}) \rightarrow{ }^{3} \mathrm{~A}_{2}(\mathrm{~F})\right)$ and $\left({ }^{3} \mathrm{~T}_{1}(\mathrm{~F}) \rightarrow{ }^{3} \mathrm{~T}_{1}(\mathrm{P})\right)$ transitions in a tetrahedral geometry (Nicholls, 1973), respectively, the complexes (16-21) show three absorption bands in the range $\left(9872-10355 \mathrm{~cm}^{-1}\right),\left(14517-18161 \mathrm{~cm}^{-1}\right)$ and $\left(24509-26315 \mathrm{~cm}^{-1}\right)$, which were assigned $\left({ }^{3} \mathrm{~A}_{2} \mathrm{~g}(\mathrm{~F}) \rightarrow{ }^{3} \mathrm{~T}_{2} \mathrm{~g}(\mathrm{~F})\right),\left({ }^{3} \mathrm{~A}_{2} \mathrm{~g}(\mathrm{~F}) \rightarrow{ }^{3} \mathrm{~T}_{1} \mathrm{~g}(\mathrm{~F})\right)$ and $\left({ }^{3} \mathrm{~A}_{2} \mathrm{~g}(\mathrm{~F}) \rightarrow{ }^{3} \mathrm{~T}_{1} \mathrm{~g}(\mathrm{P})\right.$ ) (Singh et al., 1989).

The $\mathrm{Cu}(\mathrm{II})$ complex (22) gives an absorption band at $\left(10073 \mathrm{~cm}^{-1}\right)$, which corresponds to $\left({ }^{2} \mathrm{~T}_{2} \rightarrow{ }^{2} \mathrm{E}\right)$ transition in an tetrahedral geometry (Cookson et al., 2010), the complexes (23-28) show a broad band in the region (9823-12444 $\mathrm{cm}^{-1}$ ), which was assigned to $\left({ }^{2} \mathrm{Eg} \rightarrow{ }^{2} \mathrm{~T}_{2} \mathrm{~g}\right)$ transition which may be formed from the combination of three transitions $\left({ }^{2} \mathrm{~B}_{1} \mathrm{~g} \rightarrow{ }^{2} \mathrm{~A}_{1} \mathrm{~g}\right),\left({ }^{2} \mathrm{~B}_{1} \mathrm{~g} \rightarrow{ }^{2} \mathrm{~B}_{2} \mathrm{~g}\right)$ and $\left({ }^{2} \mathrm{~B}_{1} \mathrm{~g} \rightarrow{ }^{2} \mathrm{Eg}\right)$ (Lever et al., 1984).

\section{Magnetic susceptibility measurements}

The magnetic moments of the complexes Table (1) were measured at $\left(25^{\circ} \mathrm{C}\right)$. The magnetic moments for $\mathrm{Fe}(\mathrm{II}) \mathrm{Co}$ (II)and $\mathrm{Ni}$ (II) and $\mathrm{Cu}(\mathrm{II})$ complexes (1,8,15 and 22) are (1.83-4.99 B.M) suggesting a tetrahedral geometry ( Nicholls, 1973). The low values of magnetic moments for complexes (15 and 22) are due to the antiferromagnetic interaction. The magnetic moments values of the other complexes (2-7,9-14,16-21,23-28) were in the range (1.68-5.70 B.M.) in an octahedral geometry (Nicholls, 1973). 
Table 1: Analytical and some physical properties of the prepared complexes

\begin{tabular}{|c|c|c|c|c|c|c|c|}
\hline No. & Compound & Color & m.p $\left({ }^{\circ} \mathbf{c}\right)$ & $\begin{array}{l}\text { Molar conductivity } \\
\Omega^{-1} \cdot \mathrm{cm}^{2} \cdot \mathrm{mol}^{-1}\end{array}$ & $\% \mathbf{M}$ & \%yield & $\begin{array}{l}\mu_{\text {eff }} \\
\text { B.M }\end{array}$ \\
\hline $\mathrm{L}$ & $\mathrm{C}_{5} \mathrm{H}_{10} \mathrm{NOS}_{2} \mathrm{~K}$ & White & $134-136$ & --- & --- & --- & --- \\
\hline 1 & {$\left[\mathrm{Fe}(3-\mathrm{MeoPrdtc})_{2}\right]$} & Orange & $285^{*}$ & 13.98 & $\begin{array}{l}13.70 \\
(14.54)\end{array}$ & 49 & 4.99 \\
\hline 2 & {$\left[\mathrm{Fe}(3-\mathrm{MeoPrdtc})_{2}(\mathrm{Py})_{2}\right]$} & Orange & $280^{*}$ & 22.90 & $\begin{array}{l}9.92 \\
(10.30)\end{array}$ & 70 & 5.09 \\
\hline 3 & {$\left[\mathrm{Fe}(3-\mathrm{MeoPrdtc})_{2}(\mathrm{IsoQ})_{2}\right]$} & $\begin{array}{l}\text { Light } \\
\text { brown }\end{array}$ & $270^{*}$ & 5.33 & $\begin{array}{l}8.48 \\
(9.08) \\
\end{array}$ & 52 & 5.35 \\
\hline 4 & {$\left[\mathrm{Fe}(3-\mathrm{MeoPrdtc})_{2}(\gamma-\mathrm{pico})_{2}\right]$} & Orange & $290^{*}$ & 20.20 & $\begin{array}{l}9.10 \\
(9.80) \\
\end{array}$ & 68 & 5.33 \\
\hline 5 & {$\left[\mathrm{Fe}(3-\mathrm{MeoPrdtc})_{2}(3,5-\mathrm{lut})_{2}\right]$} & Orange & $240^{*}$ & $12.02^{\cdots}$ & $\begin{array}{l}8.79 \\
(9.34)\end{array}$ & 61 & 4.96 \\
\hline 6 & {$\left[\mathrm{Fe}(3-\mathrm{MeoPrdtc})_{2}(\mathrm{en})\right]$} & Brown & $200^{*}$ & 15.72 & $\begin{array}{l}11.99 \\
(12.58)\end{array}$ & 78 & 5.16 \\
\hline 7 & $\begin{array}{l}{\left[\mathrm{Fe}(3-\mathrm{MeoPrdtc})_{2}(1,10-\right.} \\
\text { phen })]\end{array}$ & $\begin{array}{l}\text { Light } \\
\text { purple }\end{array}$ & $105-107$ & 10.50 & $\begin{array}{l}8.93 \\
(9.02)\end{array}$ & 60 & 5.70 \\
\hline 8 & {$\left[\mathrm{Co}(3-\mathrm{MeoPrdtc})_{2}\right]$} & $\begin{array}{l}\text { Dark } \\
\text { green }\end{array}$ & $146-149$ & 10.90 & $\begin{array}{l}14.73 \\
(15.22)\end{array}$ & 55 & 4.10 \\
\hline 9 & {$\left[\mathrm{Co}(3-\mathrm{MeoPrdtc})_{2}(\mathrm{Py})_{2}\right]$} & $\begin{array}{l}\text { Dark } \\
\text { brown } \\
\end{array}$ & $109-112$ & 15.40 & $\begin{array}{l}9.29 \\
(10.28)\end{array}$ & 54 & 5.1 \\
\hline 10 & {$\left[\mathrm{Co}(3-\mathrm{MeoPrdtc})_{2}(\mathrm{IsoQ})_{2}\right]$} & \begin{tabular}{|l} 
Dark \\
brown
\end{tabular} & $90^{*}$ & 6.20 & $\begin{array}{l}8.44 \\
(9.13) \\
\end{array}$ & 62 & 4.69 \\
\hline 11 & {$\left[\mathrm{Co}(3-\mathrm{MeoPrdtc})_{2}(\gamma-\text { pico })_{2}\right]$} & $\begin{array}{l}\text { Dark } \\
\text { brown }\end{array}$ & $123-127$ & 3.30 & $\begin{array}{l}8.84 \\
(10.81)\end{array}$ & 60 & 4.34 \\
\hline 12 & {$\left[\mathrm{Co}(3-\mathrm{MeoPrdtc})_{2}(3,5-\mathrm{lut})_{2}\right]$} & $\begin{array}{l}\text { Dark } \\
\text { brown }\end{array}$ & $228-231$ & 7.30 & $\begin{array}{l}8.03 \\
(9.80)\end{array}$ & 50 & 4.71 \\
\hline 13 & {$\left[\mathrm{Co}(3-\mathrm{MeoPrdtc})_{2}(\mathrm{en})\right]$} & Black & $200^{*}$ & 12.40 & $\begin{array}{l}12.83 \\
(13.18) \\
\end{array}$ & 85 & 1.93 \\
\hline 14 & $\begin{array}{l}{\left[\mathrm{Co}(3-\mathrm{MeoPrdtc})_{2}(1,10-\right.} \\
\text { phen })]\end{array}$ & \begin{tabular}{|l} 
Dark \\
brown
\end{tabular} & $97-100$ & 14.87 & $\begin{array}{l}8.38 \\
(9.48) \\
\end{array}$ & 67 & 1.77 \\
\hline 15 & {$\left[\mathrm{Ni}(3-\mathrm{MeoPrdtc})_{2}\right]$} & \begin{tabular}{|l|} 
Dark \\
green
\end{tabular} & $142-145$ & 0.90 & $\begin{array}{l}14.14 \\
(15.18)\end{array}$ & 82 & 3.20 \\
\hline 16 & {$\left[\mathrm{Ni}(3-\mathrm{MeoPrdtc})_{2}(\mathrm{Py})_{2}\right]$} & Orange & $143-146$ & 6.10 & $\begin{array}{l}10.45 \\
(10.77) \\
\end{array}$ & 50 & 2.73 \\
\hline 17 & {$\left[\mathrm{Ni}(3-\mathrm{MeoPrdtc})_{2}(\mathrm{IsoQ})_{2}\right]$} & $\begin{array}{l}\text { Dark } \\
\text { green }\end{array}$ & $132-135$ & 0.40 & $\begin{array}{l}8.60 \\
(9.10) \\
\end{array}$ & 54 & 2.99 \\
\hline 18 & {$\left[\mathrm{Ni}(3-\mathrm{MeoPrdtc})_{2}(\gamma-\mathrm{pico})_{2}\right]$} & $\begin{array}{l}\text { Dark } \\
\text { green }\end{array}$ & $133-138$ & 10.00 & $\begin{array}{l}10.08 \\
(10.25) \\
\end{array}$ & 77 & 2.77 \\
\hline 19 & {$\left[\mathrm{Ni}(3-\mathrm{MeoPrdtc})_{2}(3,5 \text {-lut })_{2}\right]$} & $\begin{array}{l}\text { Dark } \\
\text { green }\end{array}$ & $119-121$ & 14.90 & $\begin{array}{l}9.48 \\
(9.77) \\
\end{array}$ & 91 & 3.05 \\
\hline 20 & {$\left[\mathrm{Ni}(3-\mathrm{MeoPrdtc})_{2}(\mathrm{en})\right]$} & $\begin{array}{l}\text { Light } \\
\text { purple }\end{array}$ & $230^{*}$ & 12.40 & $\begin{array}{l}12.46 \\
(13.13)\end{array}$ & 62 & 2.98 \\
\hline 21 & $\begin{array}{l}{\left[\mathrm{Ni}(3-\mathrm{MeoPrdtc})_{2}(1,10-\right.} \\
\text { phen)] }\end{array}$ & \begin{tabular}{|l|} 
Dark \\
green
\end{tabular} & $142-147$ & 0.40 & $\begin{array}{l}9.05 \\
(9.44) \\
\end{array}$ & 42 & 2.36 \\
\hline 22 & {$\left[\mathrm{Cu}(3-\mathrm{MeoPrdtc})_{2}\right]$} & $\begin{array}{l}\text { Dark } \\
\text { brown }\end{array}$ & $109-112$ & 3.30 & $\begin{array}{l}15.11 \\
(16.22) \\
\end{array}$ & 73 & 1.83 \\
\hline 23 & {$\left[\mathrm{Cu}(3-\mathrm{MeoPrdtc})_{2}(\mathrm{Py})_{2}\right]$} & $\begin{array}{l}\text { Light } \\
\text { yellow }\end{array}$ & $290^{*}$ & 1.70 & $\begin{array}{l}10.93 \\
(11.56) \\
\end{array}$ & 76 & 2.06 \\
\hline 24 & {$\left[\mathrm{Cu}(3-\mathrm{MeoPrdtc})_{2}(\mathrm{IsoQ})_{2}\right]$} & $\begin{array}{l}\text { Green } \\
\text { yellow }\end{array}$ & $98-102$ & 16.80 & $\begin{array}{l}9.46 \\
(9.78) \\
\end{array}$ & 55 & 1.77 \\
\hline 25 & {$\left[\mathrm{Cu}(3-\mathrm{MeoPrdtc})_{2}(\gamma-\text { pico })_{2}\right]$} & Brown & $110-113$ & 2.30 & $\begin{array}{l}10.39 \\
(11.00)\end{array}$ & 72 & 1.68 \\
\hline
\end{tabular}


Saad E. AL-Mukhtar and Marwa Th. Aghwan

\begin{tabular}{|c|c|c|c|c|c|c|c|}
\hline No. & Compound & Color & m.p $\left({ }^{\circ} \mathrm{c}\right)$ & $\begin{array}{l}\text { Molar conductivity } \\
\Omega^{-1} \cdot \mathrm{cm}^{2} \cdot \mathrm{mol}^{-1}\end{array}$ & $\% \mathbf{M}$ & \%yield & $\begin{array}{l}\mu_{\text {eff }} \\
\text { B.M }\end{array}$ \\
\hline 26 & {$\left[\mathrm{Cu}(3-\mathrm{MeoPrdtc})_{2}(3,5-\mathrm{lut})_{2}\right]$} & Brown & 104-107 & 11.86 & 9.72 & 65 & 1.96 \\
\hline 27 & {$\left[\mathrm{Cu}(3-\mathrm{MeoPrdtc})_{2}(\mathrm{en})\right]$} & $\begin{array}{l}\text { Light } \\
\text { brown }\end{array}$ & $96-99$ & 18.60 & $\begin{array}{l}13.23 \\
(14.07)\end{array}$ & 66 & 1.69 \\
\hline 28 & $\begin{array}{l}{\left[\mathrm{Cu}(3-\mathrm{MeoPrdtc})_{2}(1,10-\right.} \\
\text { phen)] }\end{array}$ & Yellow & $99-102$ & 24.60 & $\begin{array}{l}9.39 \\
(10.14)\end{array}$ & 68 & 2.02 \\
\hline 29 & {$\left[\mathrm{Zn}(3-\mathrm{MeoPrdtc})_{2}\right]$} & White & $98-100$ & 1.10 & $\begin{array}{l}16.09 \\
(16.61)\end{array}$ & 58 & Dia \\
\hline 30 & {$\left[\mathrm{Zn}(3-\mathrm{MeoPrdtc})_{2}(\mathrm{Py})_{2}\right]$} & White & $101-104$ & 9.10 & $\begin{array}{l}11.44 \\
(11.85)\end{array}$ & 52 & Dia \\
\hline 31 & {$\left[\mathrm{Zn}(3-\mathrm{MeoPrdtc})_{2}(\mathrm{IsoQ})_{2}\right]$} & $\begin{array}{l}\text { Light } \\
\text { yellow }\end{array}$ & $210^{*}$ & 20.60 & $\begin{array}{l}8.90 \\
(10.03) \\
\end{array}$ & 53 & Dia \\
\hline 32 & {$\left[\mathrm{Zn}(3-\mathrm{MeoPrdtc})_{2}(\gamma \text {-pico })_{2}\right]$} & White & 116-119 & 14.08 & $\begin{array}{l}10.86 \\
(11.28)\end{array}$ & 69 & Dia \\
\hline 33 & {$\left[\mathrm{Zn}(3-\mathrm{MeoPrdtc})_{2}(3,5-\mathrm{lut})_{2}\right]$} & $\begin{array}{l}\text { Light } \\
\text { yellow }\end{array}$ & $101-104$ & 18.00 & $\begin{array}{l}9.83 \\
(10.76) \\
\end{array}$ & 63 & Dia \\
\hline 34 & {$\left[\mathrm{Zn}(3-\mathrm{MeoPrdtc})_{2}(\mathrm{en})\right]$} & Yellow & $124-127$ & 24.90 & $\begin{array}{l}13.96 \\
(14.41)\end{array}$ & 88 & Dia \\
\hline 35 & $\begin{array}{l}{\left[\mathrm{Zn}(3-\mathrm{MeoPrdtc})_{2}(1,10-\right.} \\
\text { phen })]\end{array}$ & $\begin{array}{l}\text { Light } \\
\text { yellow }\end{array}$ & $235^{*}$ & 25.10 & $\begin{array}{l}9.14 \\
(10.41)\end{array}$ & 66 & Dia \\
\hline
\end{tabular}

*decomposition

\section{Table 2: IR bands $\left(\mathrm{cm}^{-1}\right)$ and electronic spectral data of ligand and prepared complexes}

\begin{tabular}{|c|c|c|c|c|c|c|c|}
\hline \multirow[b]{2}{*}{ No. } & \multirow[b]{2}{*}{ Compound } & \multirow[b]{2}{*}{ UV bands $\left(\mathrm{cm}^{-1}\right)$} & \multicolumn{5}{|c|}{ IR spectra $\left(\mathrm{cm}^{-1}\right)$} \\
\hline & & & $\begin{array}{l}v(C-\cdots \\
N)\end{array}$ & $v(C-S)$ & $\begin{array}{l}v(C=N) \\
\text { ring }\end{array}$ & $v(M-N)$ & v(M-S) \\
\hline $\mathrm{L}$ & $\mathrm{C}_{5} \mathrm{H}_{10} \mathrm{NOS}_{2} \mathrm{~K}$ & --- & 1487 & 1045 & --- & --- & --- \\
\hline 1 & {$\left[\mathrm{Fe}(3-\mathrm{MeoPrdtc})_{2}\right]$} & 11180,36231 & 1540 & 983 & --- & --- & 430 \\
\hline 2 & {$\left[\mathrm{Fe}(3-\mathrm{MeoPrdtc})_{2}(\mathrm{Py})_{2}\right]$} & 10910,34695 & 1531 & 991 & 1522 & 478 & 440 \\
\hline 3 & {$\left[\mathrm{Fe}(3-\mathrm{MeoPrdtc})_{2}(\mathrm{IsoQ})_{2}\right]$} & 10100,32679 & 1509 & 1038 & 1498 & 480 & 434 \\
\hline 4 & {$\left[\mathrm{Fe}(3-\mathrm{MeoPrdtc})_{2}(\gamma-\text { pico })_{2}\right]$} & 10490,34965 & 1516 & 1020 & 1480 & 465 & 430 \\
\hline 5 & {$\left[\mathrm{Fe}(3-\mathrm{MeoPrdtc})_{2}(3,5-\mathrm{lut})_{2}\right]$} & 10514,35971 & 1513 & 1020 & 1450 & 466 & 445 \\
\hline 6 & {$\left[\mathrm{Fe}(3-\mathrm{MeoPrdtc})_{2}(\mathrm{en})\right]$} & $10641,22935,37037$ & 1518 & 997 & 1504 & 476 & 445 \\
\hline 7 & {$\left[\mathrm{Fe}(3-\mathrm{MeoPrdtc})_{2}(1,10\right.$-phen $\left.)\right]$} & 10309,32679 & 1527 & 999 & 1493 & 474 & 435 \\
\hline 8 & {$\left[\mathrm{Co}(3-\mathrm{MeoPrdtc})_{2}\right]$} & 15673,32467 & 1550 & 978 & --- & --- & 436 \\
\hline 9 & {$\left[\mathrm{Co}(3-\mathrm{MeoPrdtc})_{2}(\mathrm{Py})_{2}\right]$} & $\begin{array}{l}9803,15243,24390, \\
27777\end{array}$ & 1523 & 988 & 1483 & 523 & 432 \\
\hline 10 & {$\left[\mathrm{Co}(3-\mathrm{MeoPrdtc})_{2}(\operatorname{IsoQ})_{2}\right]$} & $\begin{array}{l}15576,20746, \\
32258,46296 \\
\end{array}$ & 1529 & 1016 & 1511 & 494 & 420 \\
\hline 11 & {$\left[\mathrm{Co}(3-\mathrm{MeoPrdtc})_{2}(\gamma-\text { pico })_{2}\right]$} & $\begin{array}{l}15625,25252, \\
28910,32467\end{array}$ & 1512 & 1028 & 1474 & 488 & 426 \\
\hline 12 & {$\left[\mathrm{Co}(3-\mathrm{MeoPrdtc})_{2}(3,5 \text {-lut })_{2}\right]$} & $\begin{array}{l}15384,24390, \\
27777,31645 \\
\end{array}$ & 1512 & 1008 & 1444 & 481 & 423 \\
\hline 13 & {$\left[\mathrm{Co}(3-\mathrm{MeoPrdtc})_{2}(\mathrm{en})\right]$} & $\begin{array}{l}9700,16891,25000, \\
29761,32894\end{array}$ & 1535 & 991 & 1490 & 475 & 428 \\
\hline 14 & {$\left[\mathrm{Co}(3-\mathrm{MeoPrdtc})_{2}(1,10-\right.$ phen $\left.)\right]$} & $\begin{array}{l}9842,15243,24039, \\
30120\end{array}$ & 1533 & 996 & 1489 & 491 & 426 \\
\hline 15 & {$\left[\mathrm{Ni}(3-\mathrm{MeoPrdtc})_{2}\right]$} & $\begin{array}{l}10066,13022 \\
20661,25510\end{array}$ & 1545 & 966 & --- & --- & 440 \\
\hline
\end{tabular}




\begin{tabular}{|c|c|c|c|c|c|c|c|}
\hline \multirow[b]{2}{*}{ No. } & \multirow[b]{2}{*}{ Compound } & \multirow[b]{2}{*}{ UV bands $\left(\mathrm{cm}^{-1}\right)$} & \multicolumn{5}{|c|}{ IR spectra $\left(\mathrm{cm}^{-1}\right)$} \\
\hline & & & $\begin{array}{l}\mathrm{v}(\mathrm{C}-\cdots \\
\mathrm{N})\end{array}$ & $v(C-S)$ & $\begin{array}{l}v(C=N) \\
\text { ring }\end{array}$ & $v(M-N)$ & $v(\mathrm{M}-\mathrm{S})$ \\
\hline 16 & {$\left[\mathrm{Ni}(3-\mathrm{MeoPrdtc})_{2}(\mathrm{Py})_{2}\right]$} & $\begin{array}{l}10163,16078, \\
25510,29585\end{array}$ & 1500 & 968 & 1441 & 478 & 430 \\
\hline 17 & {$\left[\mathrm{Ni}(3-\mathrm{MeoPrdtc})_{2}(\mathrm{IsoQ})_{2}\right]$} & $\begin{array}{l}10355,18161, \\
24509,30303\end{array}$ & 1507 & 1030 & 1473 & 476 & 433 \\
\hline 18 & {$\left[\mathrm{Ni}(3-\mathrm{MeoPrdtc})_{2}(\gamma-\mathrm{pico})_{2}\right]$} & $\begin{array}{l}9872,16049,24752, \\
49019\end{array}$ & 1501 & 976 & 1490 & 474 & 425 \\
\hline 19 & {$\left[\mathrm{Ni}(3-\mathrm{MeoPrdtc})_{2}(3,5-\mathrm{lut})_{2}\right]$} & $\begin{array}{l}9957,18118,25125, \\
29585\end{array}$ & 1528 & 968 & 1487 & 480 & 429 \\
\hline 20 & {$\left[\mathrm{Ni}(3-\mathrm{MeoPrdtc})_{2}(\mathrm{en})\right]$} & $\begin{array}{l}10163,14517, \\
26315,29339 \\
\end{array}$ & 1511 & 995 & 1458 & 499 & 441 \\
\hline 21 & {$\left[\mathrm{Ni}(3-\mathrm{MeoPrdtc})_{2}(1,10-\right.$ phen $\left.)\right]$} & $\begin{array}{l}9990,15886,25125, \\
29339\end{array}$ & 1545 & 966 & 1490 & 474 & 439 \\
\hline 22 & {$\left[\mathrm{Cu}(3-\mathrm{MeoPrdtc})_{2}\right]$} & 10073,33333 & 1500 & 939 & --- & --- & 439 \\
\hline 23 & {$\left[\mathrm{Cu}(3-\mathrm{MeoPrdtc})_{2}(\mathrm{Py})_{2}\right]$} & $\begin{array}{l}12444,17985, \\
36764\end{array}$ & 1493 & 951 & 1478 & 476 & 426 \\
\hline 24 & {$\left[\mathrm{Cu}(3-\mathrm{MeoPrdtc})_{2}(\mathrm{IsoQ})_{2}\right]$} & 10917,28409 & 1500 & 943 & 1450 & 482 & 436 \\
\hline 25 & {$\left[\mathrm{Cu}(3-\mathrm{MeoPrdtc})_{2}(\gamma-\mathrm{pico})_{2}\right]$} & $9823,23474,27472$ & 1498 & 984 & 1444 & 465 & 422 \\
\hline 26 & {$\left[\mathrm{Cu}(3-\mathrm{MeoPrdtc})_{2}(3,5-\mathrm{lut})_{2}\right]$} & $9842,26595,31055$ & 1490 & 941 & 1450 & 465 & 438 \\
\hline 27 & {$\left[\mathrm{Cu}(3-\mathrm{MeoPrdtc})_{2}(\mathrm{en})\right]$} & $\begin{array}{l}12297,31645, \\
35211\end{array}$ & 1492 & 1007 & 1462 & 476 & 439 \\
\hline 28 & {$\left[\mathrm{Cu}(3-\mathrm{MeoPrdtc})_{2}(1,10\right.$-phen $\left.)\right]$} & 24038,32894 & 1498 & 945 & 1465 & 492 & 438 \\
\hline 29 & {$\left[\mathrm{Zn}(3-\mathrm{MeoPrdtc})_{2}\right]$} & $\begin{array}{c}-- \\
\end{array}$ & 1516 & 964 & --- & --- & 431 \\
\hline 30 & {$\left[\mathrm{Zn}(3-\mathrm{MeoPrdtc})_{2}(\mathrm{Py})_{2}\right]$} & --- & 1510 & 1036 & 1452 & 488 & 430 \\
\hline 31 & {$\left[\mathrm{Zn}(3-\mathrm{MeoPrdtc})_{2}(\mathrm{IsoQ})_{2}\right]$} & --- & 1496 & 1017 & 1486 & 484 & 418 \\
\hline 32 & {$\left[\mathrm{Zn}(3-\mathrm{MeoPrdtc})_{2}(\gamma-\mathrm{Pico})_{2}\right]$} & --- & 1509 & 1009 & 1477 & 494 & 424 \\
\hline 33 & {$\left[\mathrm{Zn}(3-\mathrm{MeoPrdtc})_{2}(3,5-\mathrm{lut})_{2}\right]$} & --- & 1511 & 991 & 1446 & 489 & 420 \\
\hline 34 & {$\left[\mathrm{Zn}(3-\mathrm{MeoPrdtc})_{2}(\mathrm{en})\right]$} & --- & 1506 & 973 & 1473 & 482 & 418 \\
\hline 35 & {$\left[\mathrm{Zn}(3-\mathrm{MeoPrdtc})_{2}(1,10\right.$-phen $\left.)\right]$} & ---- & 1508 & 997 & 1489 & 478 & 419 \\
\hline
\end{tabular}

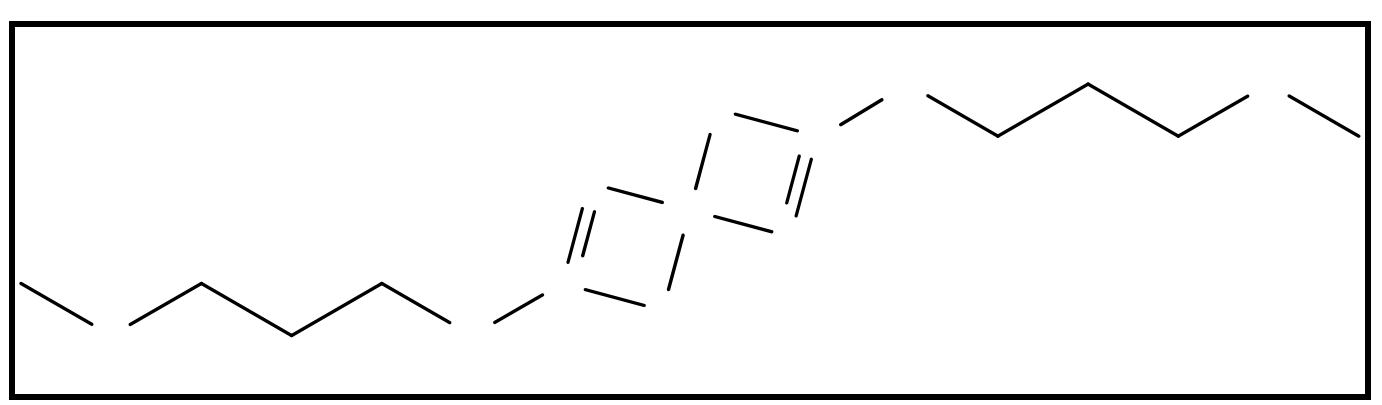

(a) 


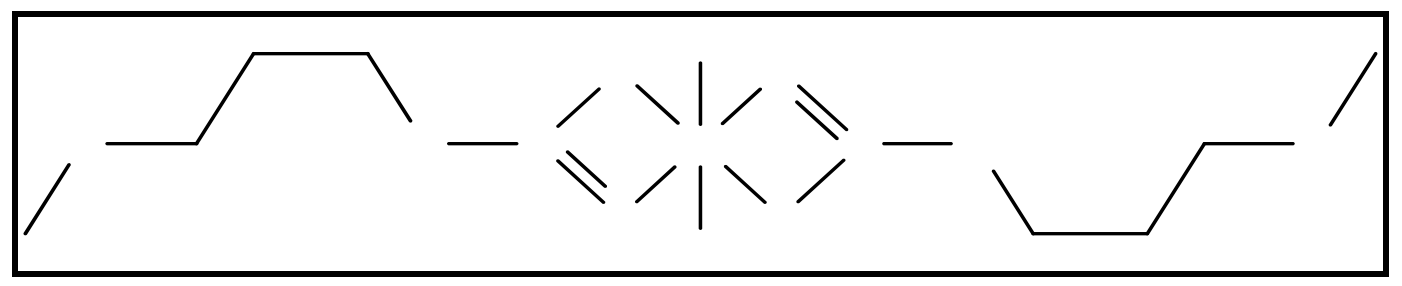

(b)

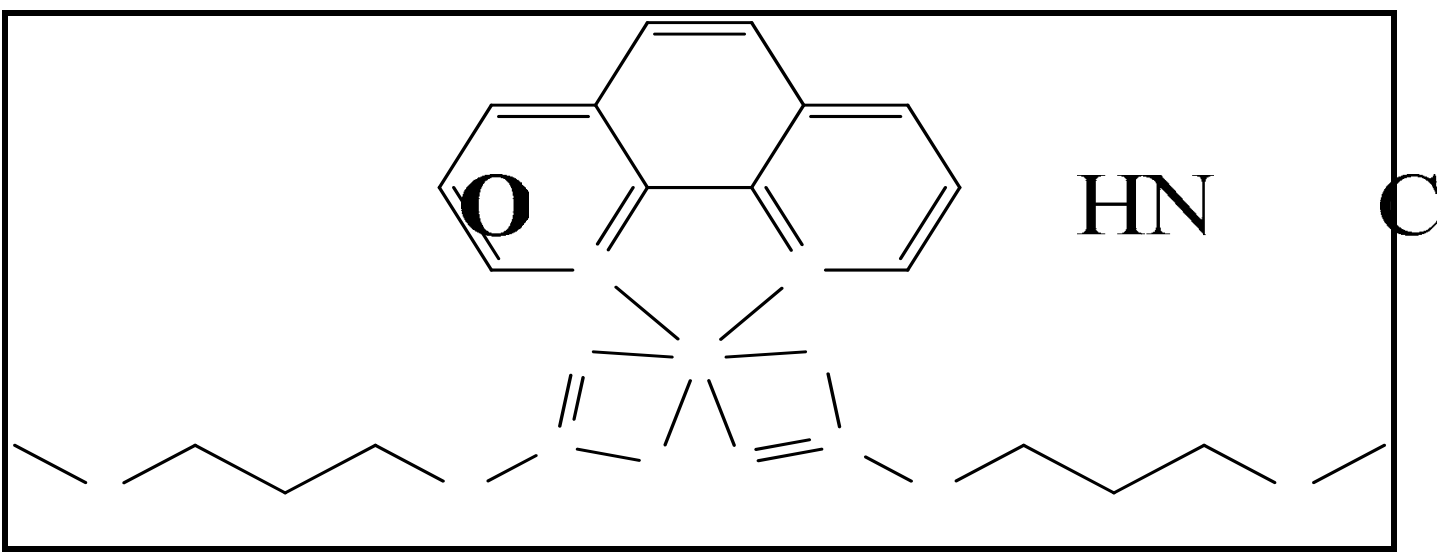

$\mathbf{S}$

(c)

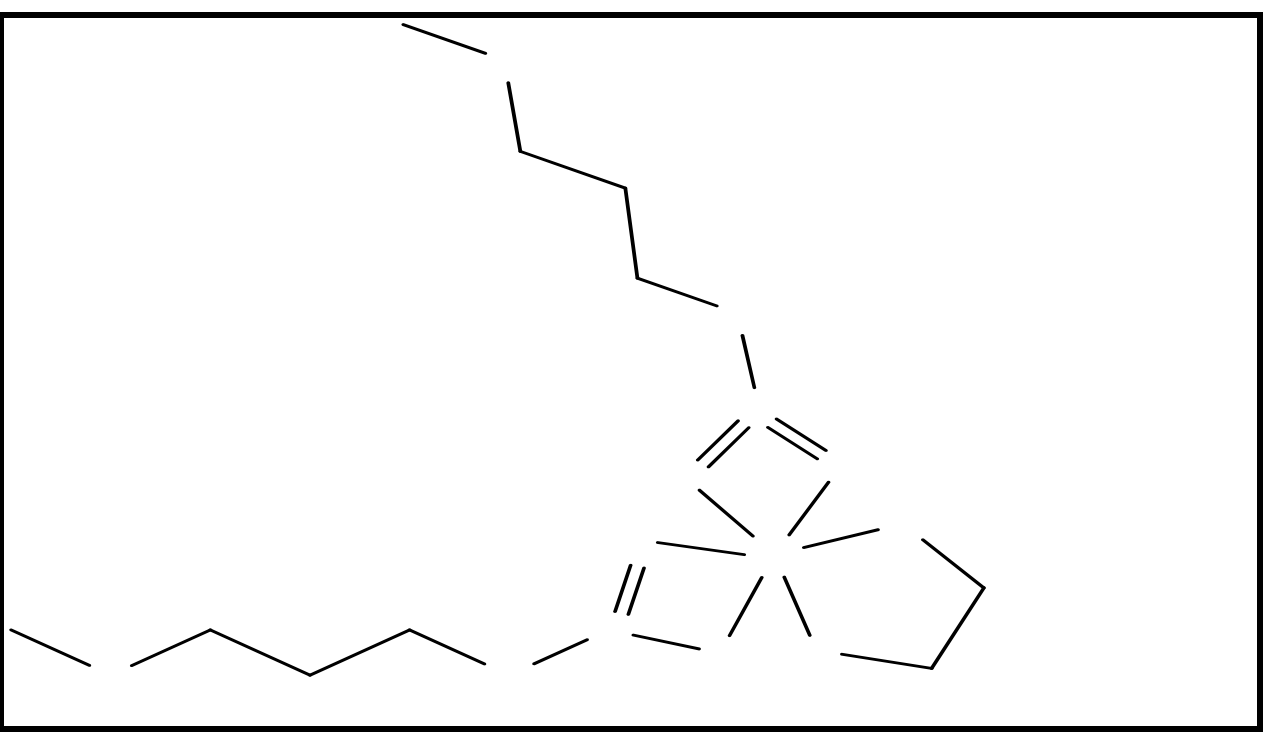

(d)

N

Fig. 1: Suggest structures (a) complexes $[1,8,15,22,29]$, (b) complexes $[2-5,9-12,16-$ 19, 23-26, 30-33], (c) complexes $[6,13,20,27,34]$, (d) complexes $[7,14,21,28,35]$

\section{REFERENCES}

Cookson, J.; Emma, A.L.E.; John, P.M.; Christopher, J.S.; Rowena, L.P. Andrew, R.C.; Michael, G.B.D.; Paul, D. (2010). Metal directed assembly of latge dinuclear copper(II) dithiocarbamate Macrocyclic complexes. Inorg. Chem. Acta., 363, 11951203.

Cotero-Villegas, A.M.; García, P.G.; Höplf-Bachner, H.; Pérez-Redondo, M.C.; MartínezSalas, P.; López-Cardoso, M.; Olivares, R. C. (2010). Synthesis and spectroscopic characterization of six new monosubstituted dithiocarbamate compounds containing 
organotellurium(IV) heterocycles: Single-crystal structures of $\mathrm{C}_{5} \mathrm{H}_{10} \mathrm{TeI}\left[\mathrm{S}_{2} \mathrm{CN}\left(\mathrm{CH}_{2} \mathrm{CH}_{3}\right)_{2}\right], \mathrm{C}_{4} \mathrm{H}_{8} \mathrm{TeI}\left[\mathrm{S}_{2} \mathrm{CN}\left(\mathrm{CH}_{2} \mathrm{CH}_{2}\right)_{2}\right],{ }_{4} \mathrm{H}_{8} \mathrm{OTeI}\left[\mathrm{S}_{2} \mathrm{CN}\left(\mathrm{CH}_{2} \mathrm{CH}_{2}\right)_{2}\right]$ and $\mathrm{C}_{5} \mathrm{H}_{10} \mathrm{TeI}\left[\mathrm{S}_{2} \mathrm{CN}\left(\mathrm{CH}_{2} \mathrm{CH}_{2}\right)_{2}\right]$. J. Organometallic Chem. 695, 1246-1252.

Coucouvanis, D.; Fackler, J.P. (1967). Square-planar sulfur complexes.VI. reactions of bases with xanthate, dithiocarbamates, and dithiolates of nickel(II). J. Inorg. Chem., 6, 2047-2053.

De Lima, G.M.; Menezes, D.C.; Cavalcanti, C.A.; Dos Santos, J.A.F.; Ferreira, I.P.; Paniago, E.B.; Wardell, J.L.; Wardell, S. M.S.V.; Krambrock, K.; Mendes, I.C.; Beraldo, H. (2011). Synthesis, characterisation and biological aspects of copper(II) dithiocarbamate complexes, $\left[\mathrm{Cu}\left\{\mathrm{S}_{2} \mathrm{CNR}\left(\mathrm{CH}_{2} \mathrm{CH}_{2} \mathrm{OH}\right)\right\}_{2}\right]$, ( $\mathrm{R}=\mathrm{Me}$, Et, $\mathrm{Pr}$ and $\left.\mathrm{CH}_{2} \mathrm{CH}_{2} \mathrm{OH}\right)$. J. Molecular Struct., 988, 1-8.

Geary, W.J. (1971). The use of conductivity measurements in organic solvents for characterization of coordination compounds. Coord. Chem. Rev. 7, 8-122.

Golcu, A. (2006). Transition metal complexes of propranolol dithiocarbamate: synthesis, characterization, analytical properties and biological activity. Trans. Metal. Chem. 31, 405-412.

Holah, D.G.; Murphy, C.N. (1972). Transition metal complexes involving ionic xanthates, Inorg. Nucl. Chem., 8,1069-1072.

Leka, Z.B.; Leovac, V.M.; Luki, S.; Sabo, T.J.; Trifunovi, S.R. ; Szécsényi, K.M. (2006). Synthesis and physico-chemical characterization of new dithiocarbamato ligandL and its complexes copper(II), nickel(II) and palladium(II). J. Therm. Analy. Calor., 83(3), 687-691.

Lever, A.B.P. (1984). "Inorganic Electronic Spectroscopy". 2nd ed., Elsevier, Amsterdam, $357 \mathrm{p}$.

Martell, A.E. (1971). "Coordination Chemistry". Van Nostrand Reinhold, New York., 1 p.

Montagner, D.; Marzano, C.; Gandin, V. (2011). Synthesis, characterization and cytotoxic activity of palladium (II)dithiocarbamate complexes with a x-diamines. Inorg. Chem. Acta., 376, 574-580.

Nicholls, D. (1973). "The Chemistry of Iron, Cobalt and Nickel". Pergamon press, Oxford, 1st ed., pp.1037, 1087, 1088,1090, 1091,1093, 1151,1154.

Onwudiwe, D.C.; Ajibade, P.A. (2010). Synthesis and characterization of metal complexes of N-alkyl-N-phenyl dithiocarbamates. Polyhedron., 29,1431-1436.

Onwudiwe, D.C.; Ajibade, P.A. (2011). Synthesis and Crystal Structure of Bis (N-alkyl-Nphenyl dithiocarbamato) mercury(II). J. Chem. Crystallogr., 41, 980-985.

Sarwar, M.; Ahmad, S.; Ahmad, S.; Ali, S.; Ahmed, S.A. (2007). Copper(II) complexes of pyrrolidine dithiocarbamate. Trans. Met. Chem., 32,199-203.

Serrano, J.L.; Garci'a, L.; Pe'rez, J.; Pe'rez, E.; Sa'nchez, G.; Garcı'a, J.; Lo'pez, G.; Garc1'a, G.; Molins, E. (2003). New dithiocarbamate and xanthate complexes of nickel(II)with iminophosphines. Inorg .Chem. Acta., 355, 33-40.

Shaheen, F.; Badshah, A.; Gielen, M.; Dusek, M.; Fejfarova, K.; De Vos, D.; Mirza, B. (2007). Synthesis, characterization, antibacterial and cytotoxic activity of new palladium(II) complexes with dithiocarbamate ligands: X-ray structure of bis(dibenzyl-1-S: Ś-dithiocarbamato) Pd(II). J. Organomet. Chem. 692, 3019-3026. 
Siddiqi, K.S.; Nishat, N. (2000). Synthesis and characterization of succinimide and phthalimide dithiocarbamate and their complexes with some transition metal ions. Synth. Reac. Inorg. Met-Org. Chem., 30(8), 1505 -1518.

Singh, N.; Singh, N.K.; Kaw, C. (1989). Synthetic and spectroscopic studies of xanthatebridged heterobimetallic complexes containing diamagnetic and paramagnetic metal ions. Bull. Chem. Soc. (Jpn.), 62, 3328-3333.

Srinivasan, N.; Valarmathi, P.; Thirumaran, S.; Ciattini, S. (2010). Synthesis and spectral studies on $\mathrm{NiS}_{4}, \mathrm{NiS}_{2} \mathrm{PN}, \mathrm{NiS}_{2} \mathrm{P}_{2}$ chromophores: Single-crystal X-ray structure of $\left[\mathrm{Ni}(\mathrm{dbpdtc})_{2}\right]$ (dbpdtc=benzyl(4-(benzylamino)phenyl)dithiocarbamate). Trans Met Chem., 35, 815-819.

Rathore, H.S.; Varshney, G.; Mojumdar, S.C.; Saleh, M.T. (2007). Synthesis, characterization and fungicidal activity of zinc diethyldithIocarbamate and phosphate. Ther. Anal. Calorim, 90 (3), 681-686.

Raya, I.; Baba, I. ; Yamin, B.M. (2006). New mixed ligands complexes of samarium(III) with dithiocarbamates and 1,10-phenanthroline. Malaysia. J. Anal. Sci., 10(1), 93-98. 